\title{
Identification of a novel protein encoded by third conserved gene within RAG locus
}

\author{
Monika Kasztura ${ }^{\star}$, Arkadiusz Miazek $^{\star}$, Małgorzata Cebrat and Paweł Kisielow $^{\bowtie}$ \\ Laboratory of Transgenesis and Lymphocyte Biology, Department of Tumor Immunology, Institute of \\ Immunology and Experimental Therapy, Polish Academy of Sciences, Wroctaw, Poland
}

Received: 30 January, 2009; revised: 02 March, 2009; accepted: 13 March, 2009

available on-line: 17 March, 2009

\begin{abstract}
Recently, a third evolutionarily conserved gene, $N W C$, was discovered within the recombination activating gene (RAG) locus, known to contain the RAG1 and RAG2 genes. Here, we identify and characterize the murine endogenous NWC protein which has no homology to any known protein and is ubiquitously expressed. In the cell, the NWC protein which has been suggested to function as a transcriptional repressor, is found in the cytoplasm as well as in the nucleus.
\end{abstract}

Keywords: RAG locus, evolutionarily conserved proteins, lymphocytes, T cells

\section{INTRODUCTION}

Recombination activating gene $(R A G) 1$ and 2, two closely linked, evolutionarily conserved lymphocyte specific genes, encode transposase, an essential component of the $\mathrm{V}(\mathrm{D}) \mathrm{J}$ recombinase which is responsible for the generation of diversity of antigen receptors on $\mathrm{T}$ and $\mathrm{B}$ lymphocytes (Schatz \& Baltimore, 2004). It is believed that the acquisition of RAG genes represented a turning point in evolution of vertebrates, allowing for the development of lymphocytes and adaptive immunity (Thompson, 1995). NWC is a third evolutionarily conserved gene, recently identified within the RAG locus (Cebrat et al., 2005). In contrast to $R A G$ genes, NWC is ubiquitously expressed but in lymphocytes, unlike in non-lymphocytes, it is subject to RAG1-dependent, lymphocyte specific regulation (Cebrat et al., 2005). In non-lymphocytes, it is controlled by its own, RAG2 intragenic promoter, which in lymphocytes is inactivated due to methylation (Cebrat et al., 2008). The predicted amino-acid sequence of NWC protein has no significant homology to any known protein and contains three highly conserved regions with identical sequence motifs that had not been previously described (Cebrat et al., 2005).

The above-mentioned observations suggest that NWC could play an important role in evolution and in development of lymphocytes (Kisielow et al., 2008). Unfortunately, the function of the NWC is not yet known and until now the endogenous protein product of NWC gene has not been identified. Very recently, a protein encoded by cDNA representing a splice variant of the human ortholog of the NWC gene was identified and preliminary findings suggest that it has properties of a transcriptional repressor, but attempts to demonstrate expression of this protein from the endogenous gene have failed (Hong et al., 2008).

Here we identify endogenous NWC protein in the mouse and show that it has a very wide tissue distribution. In the cell it can be found both in the cytoplasm and in the nucleus.

${ }^{\square}$ Corresponding author: P. Kisielow, Laboratory of Transgenesis and Lymphocyte Biology, Department of Tumor Immunology, Institute of Immunology and Experimental Therapy, Polish Academy of Sciences, Wrocław, Poland; tel.: (48) 71 3̧70 9952; fax: (48) 71337 1382; e-mail: kisielow@iitd.pan.wroc.pl

These authors contributed equally to this work.

Abbreviations: cDNA, complementary DNA; ECL, enhanced chemiluminescence; ELISA, enzyme-linked immunosorbent assay; MS, mass spectroscopy; PAGE, polyacrylamide gel electrophoresis; PCR, polymerase chain recation; RT-PCR, reverse transcriptase polymerase chain reaction; SDS, sodium dodecyl sulfate. 


\section{MATERIAL AND METHODS}

Cells. Murine cell lines RPC-5, P388, YAC-1, EL4 (lymphomas), 40E1 (pre-B cell), VL3-3M2 and HB10 (thymomas), NIH3T3 (fibroblast) and STO (embryonic fibroblast) were grown in culture media supplemented with 10\% FBS, 2 mM L-glutamine, $100 \mathrm{U}$ penicillin/streptomycin, $50 \mu \mathrm{M} \beta$-mercaptoethanol. $\mathrm{CD} 4^{+} \mathrm{CD} 8^{+}$thymocytes and mature $\mathrm{CD} 4^{-}$ $\mathrm{CD}^{+}$and $\mathrm{CD}^{+} \mathrm{CD}^{-}$splenic $\mathrm{T}$ cells from 6-week old C57BL/6 mice were purified using a FACS-Aria cell sorter (Beckton-Dickinson). The purity of sorted cell populations exceeded $99 \%$.

Expression vectors. To obtain a recombinant NWC-His tag protein-expressing vector (pQE-60NWC) PCR amplified cDNA fragment containing NWC open reading frame was cloned into the NcoI/ BamHI sites of pQE-60 vector (Qiagen). To obtain a mammalian NWC expression vector, (pCI-NeoNWC), PCR-amplified NWC cDNA was cloned into EcoRI/SmaI sites of pCI-Neo vector (Promega). After cloning, the NWC cDNA sequence and the in frame insertions were verified by sequencing.

Cell transfection. EL4 cells were stably transfected with pCINeo-NWC or empty pCINeo vectors (Promega) using Metafectene Pro reagent (Biontex) according to manufacturer's recommendations and selected for 2 weeks with G418 $(500 \mu \mathrm{g} / \mathrm{ml})$. G418resistant cells were cloned by limiting dilution.

Recombinant NWC protein. Expression of recombinant His-tagged NWC protein in Escherichia coli strain BL21(DE3)pLysS (Promega) transformed with pQE-60-NWC was induced by addition of $1 \mathrm{mM}$ isopropyl $\beta$-D-1-thiogalctopyranoside (IPTG). Bacteria were sonicated in lysis buffer $(50 \mathrm{mM}$ phosphate $\mathrm{pH}$ 8.0, $300 \mathrm{mM} \mathrm{NaCl}, 10 \mathrm{mM}$ imidazole) and recombinant protein was purified using Ni-NTAHis-Bind (Novagen) resins (Wisniewski et al., 2008) according to manufacturer's recommendations.

Monoclonal anti-NWC antibody (MoM12). Wistar rats were immunized subcutaneously three times with recombinant His-tagged NWC protein (200 $\mu \mathrm{g}$ per injection), in incomplete Freund's adjuvant. Two days after last injection, spleen cells were mixed with SP2/0 myeloma at a 5:1 ratio and fused with PEG 1500 (Sigma). Hybridomas were grown and selected as described by Koehler and Milstein (1975). Supernatants from growing hybridoma were screened for the presence of antibodies recognizing NWC-His using ELISA. Hybridomas secreting such antibodies were subcloned two times by limiting dilution.

Fractionation of cell lysates. Cells and tissues (tissues were first mechanically disrupted using a Polytron homogenizer (Glen Mills Inc.)) were lysed on ice in $1 \%$ Nonidet P-40 in buffer (50 mM Hepes, $\mathrm{pH}$ 7.2, $150 \mathrm{~nm} \mathrm{NaCl}, 0.5 \%$ sodium deoxycholate,
$0.1 \%$ SDS, $1 \times$ Complete protease inhibitors, Roche) for $30 \mathrm{~min}$ followed by sonication. Insoluble fragments were removed by centrifugation. For analysis by $2 \mathrm{D}$ electrophoresis protein lysates were fractionated with increasing concentration of ammonium sulfate. The fraction collected between 30 and $40 \%$ of salt saturation was centrifuged, dissolved in $50 \mathrm{mM}$ acetate buffer pH 5.5 and dialyzed. Precipitated proteins were removed by centrifugation and supernatant was purified on a DE23 column. The fraction eluted with $300 \mathrm{mM} \mathrm{NaCl}$ was enriched in a protein recognized by the MoM12 antibody. This fraction was used for further characterization by $2 \mathrm{D}$ electrophoresis. To obtain the cytoplasmic protein fraction, cells were lysed in buffer A (25 mM Hepes, $\mathrm{pH} 7.4$, $10 \mathrm{mM} \mathrm{KCl}, 150 \mathrm{mM} \mathrm{NaCl}, 0.2 \mathrm{mM}$ EDTA, $1 \%$ Triton X-100, $1 \times$ Complete protease inhibitors (Roche)) for $30 \mathrm{~min}$ and nuclei were removed by centrifugation at $600 \times g$ for $10 \mathrm{~min}$. To obtain the nuclear protein fraction, the pellet was washed 3 times with buffer A and sonicated in buffer B (25 mM Hepes, $\mathrm{pH}$ 7.4, $10 \mathrm{mM} \mathrm{KCl}, 150 \mathrm{mM} \mathrm{NaCl}, 0.2 \mathrm{mM}$ EDTA, $20 \%$ glycerol $1 \times$ Complete protease inhibitors). Protein concentration was determined using the BCA assay (Pierce). Possible cross-contamination of separated cytoplasmic and nuclear protein fractions was monitored by Western blotting using Raf-1-specific antibody (C-20, Santa Cruz - a marker of cytoplasmic fraction) and histone $\mathrm{H} 3$-specific antibody ( $\mathrm{Ab}$ cam - a marker of nuclear fraction).

2D gel electrophoresis. For two-dimensional electrophoresis (isoelectrofocusing/PAGE), proteins precipitated using methanol/acetone solution were solubilized in IEF sample buffer $(9.5 \mathrm{M}$ urea, $2 \%$ Triton X-100, 5\% 2-mercaptoethanol, 2\% ampholytes $\mathrm{pH} 3-10$ and $0.5 \% \mathrm{pH} 5-7)$ and electrophoresed in a Mini-Protean II 2-D Cell (Bio-Rad) according to manufacturer's instructions. For the second dimension electrophoresis, gel rods were applied to the top of $12.5 \%$ SDS/polyacrylamide gels. Gels were stained with a silver staining kit (Page-Silver, Fermentas) or transferred to PVDF membranes for Western blotting.

Western blotting. Proteins were electro-transferred to PVDF membranes (Millipore) in Towbin buffer (25 mM Tris, $192 \mathrm{mM}$ glycine) supplemented with $10 \%$ methanol. The membranes were blocked in 5\% skimmed milk overnight and incubated with primary antibody (MoM12, anti-Raf-1, anti-H3 or anti-actin (C-11, Santa Cruz)) for $60 \mathrm{~min}$. After washing the membranes were incubated for $30 \mathrm{~min}$ with secondary horseradish-conjugated anti-rat (Sigma), anti-goat (Santa Cruz) or anti-rabbit IgG antibody and after washing developed by enhanced chemiluminescence (ECL) detection system (Roche).

Real-time RT-PCR. Total RNA was extracted using TRIzol reagent (Invitrogen) according to 
the manufacturer's instructions. Three micrograms of RNA was reverse transcribed with Omniscript reverse transcriptase (Qiagen) at $37^{\circ} \mathrm{C}$ for $1 \mathrm{~h}$ with $100 \mathrm{ng}$ of random hexamers (Amersham Pharmacia). Real-time RT-PCR was performed with the aid of a DNA Engine Opticon 2 apparatus (MJ Research) using the Quantitect SYBR Green PCR Kit (Qiagen). The cycling conditions were: $30 \mathrm{~s}$ at $94^{\circ} \mathrm{C}, 30 \mathrm{~s}$ at $52^{\circ} \mathrm{C}$ and $1 \mathrm{~min}$ at $72^{\circ} \mathrm{C}$. Standard curves generated by amplification of 5-fold serial dilutions of cDNA template were plotted for Nwc and Hprt transcripts. The Nwc expression values were normalized to Hprt. The following primer pairs were used: Nwc: 5'-GGGAGTTCCTTTCAAGGTGATATGACC-3' (forward), 5'-GGATCATAAGATGGAACGCTGGTGC-3' (reverse); Hprt: 5'-GCTGGTGAAAAGGACCTCT-3' (forward), 5'-CACAGGACTAGAACACCTGC-3' (reverse).

\section{RESULTS AND DISCUSSION}

\section{Monoclonal antibody against recombinant NWC protein identifies endogenous mouse protein}

Long non-coding RNAs are now recognized as being four times as abundant as protein-coding genes (Kapranov et al., 2007). It was therefore important to establish whether NWC, a third evolutionarily conserved gene identified within the $R A G$ locus (Cebrat et al., 2005), encodes a protein.

The recombinant His-tagged NWC protein was expressed in E. coli and purified using Ni-NTA column. Two proteins, one corresponding to a fulllength NWC protein and the second to its N-terminally truncated form (as established by MS sequencing; not shown) were obtained which under conditions of SDS/PAGE electrophoresis migrated as $37 \mathrm{kDa}$ and $25 \mathrm{kDa}$ species, respectively (not shown). The discrepancy between the predicted $(26 \mathrm{kDa})$ and

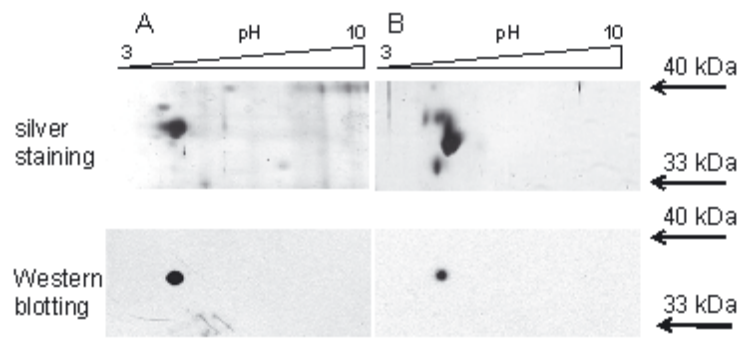

Figure 1. Identification of endogenous murine protein by MoM12 monoclonal antibody specific for recombinant NWC protein.

Recombinant NWC protein (A) or partially purified protein lysate of HB10 murine cell line (B) was subjected to 2D gel electrophoresis and analysed by silver staining and Western blotting using MoM12 antibody.
A

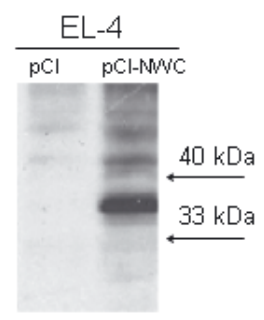

$\mathrm{B}$

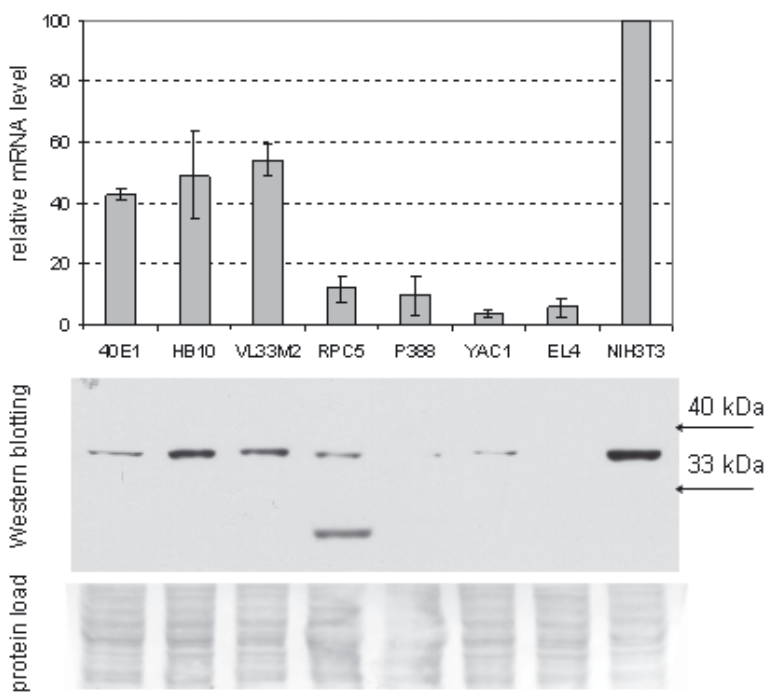

Figure 2. Endogenous murine protein identified by MoM12 antibody is encoded by NWC.

A. Murine $\mathrm{T}$ cell lymphoma EL4 was transfected with pCI-Neo-NWC (cDNA-NWC) or empty pCI-Neo vector and cell lysates were analysed by Western blotting using MoM12. B. The levels of NWC transcript and of the protein identified by MoM12 antibody in indicated cell lines were assessed using real-time PCR and Western blotting, respectively. Ponceau staining of the PVDF membrane is shown as a control of the protein load in Western blotting experiment.

observed $(37 \mathrm{kDa})$ approximate molecular mass of the full-length NWC protein is probably due to its high negative charge which can cause retardation of migration in denaturing polyacrylamide gel electrophoresis (SDS/PAGE) as is the case, for example, of LAT protein (Zhang et al., 1998). A rat monoclonal antibody (MoM12) against full-length recombinant NWC protein was obtained and used to identify endogenous NWC protein in mouse tissues. As shown in Fig. 1, MoM12 detects an endogenous mouse protein with a similar migration pattern in 2D gel electrophoresis to that of recombinant NWC protein. Both proteins recognized by MoM12 are acidic proteins of $37 \mathrm{kDa}$. Figure 2A shows that transfection of mouse lymphoid cell line EL4 with NWC cDNA results in a very strong up-regulation of the level of the protein recognized by MoM12. The level of the MoM12-reactive protein in non-transfected EL4 cells 


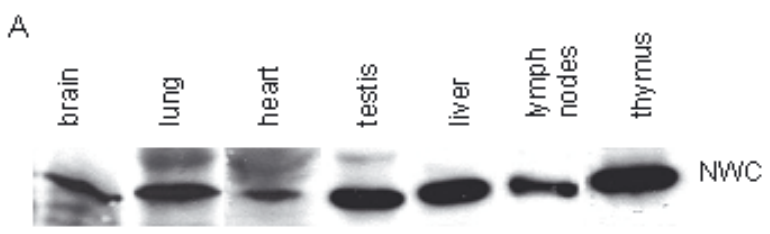

$\mathrm{B}$

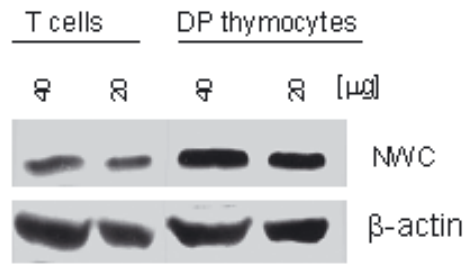

Figure 3. Expression of NWC protein in different tissues, including mature lymphocytes.

Protein extracts of indicated tissues (A) or indicated amounts of total cellular proteins from sorted CD4 and CD8 splenic $\mathrm{T}$ cells and $\mathrm{CD}^{+} 8^{+}$thymocytes ( $>99 \%$ pure) (B) were analysed by Western blotting using MoM12 antibody. Indicated tissues were analysed in 3 to 6 independent experiments.

is very low, in many experiments hardly detectable. Thus, the above results demonstrate that NWC encodes a protein in eukaryotic cells and confirms the specificity of MoM12 for the endogenous NWC protein. The close correlation between the level of NWC RNA and the amount of the endogenous protein detected by MoM12 (Fig. 2B) provides further support for the conclusion that the identified protein is indeed encoded by NWC.

Tissue distribution of NWC protein

Figure 3A shows that NWC protein is expressed in all examined tissues. However, the detectability in some tissues (e.g. brain) varies strongly between experiments and the reason for such variablity is not clear. Testis and thymus are the only tissues in which the NWC protein is consistently detected at a high level. Figure 3B shows that NWC protein can be also detected in purified mature lymphocytes. In the context of our initial observations concerning the level of NWC transcription (Cebrat et al., 2005), the finding that the NWC protein is easily and consistently detectable in mature lymphocytes was surprising. Originally, the very low level of NWC transcript observed in RAG-negative lymphocytes was interpreted as resulting from contaminating cells (Cebrat et al., 2005). The present results, using highly purified populations of peripheral $\mathrm{T}$ cells, strongly suggest that NWC can be transcribed and translated in mature lymphocytes, albeit at a relatively low level. This is consistent with our recent observation (M. Cebrat, unpublished) that in a small

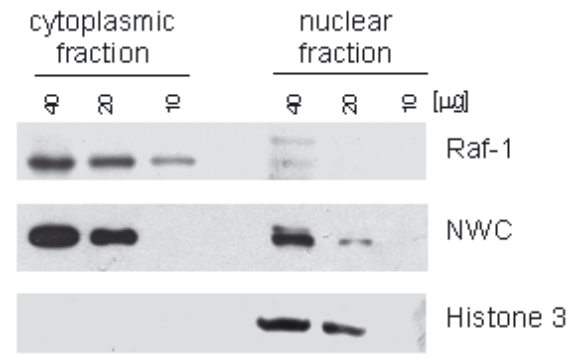

Figure 4. Intracellular distribution of NWC protein. Protein extracts of cytoplasmic and nuclear fractions of STO cells were analysed by Western blotting using indicated antibodies. Possible contamination of cytoplasmic fraction by nuclear proteins and vice versa was monitored by anti-H3 and anti-Raf-1 antibodies, respectively. Inverse correlation between the strength of NWC to Raf-1 signal (quantified by protein dilution) indicates that the detection of NWC protein in nuclear fraction is not due to the contamination with cytoplasmic fraction.

fraction of mature lymphocytes the NWC promoter is not methylated, which could be responsible for the observed protein expression level.

\section{Intracellular distribution of NWC protein}

In order to determine the intracellular localization of NWC protein, the nuclear and cytoplasmic fractions were separated and Western blotting using MoM12 was performed.

As shown in Fig. 4, NWC protein can be detected in both fractions. The extent of possible contamination of the nuclear fraction by cytoplasmic proteins and vice versa was monitored by the presence of Raf- 1 proteins and histone $\mathrm{H} 3$, respectively. The very week signal produced by the antiRaf- 1 antibody in the nuclear fraction indicates that some contamination with cytoplasmic proteins has occurred but this cannot explain the presence of NWC in this fraction because the strength of the NWC to Raf-1 signal (quantified by dilution) shows a reverse correlation to that observed in the cytoplasmic fraction. We conclude that the intracellular localization of NWC protein is not restricted to the nucleus or to the cytoplasm. Considering the possibility that NWC protein functions as a transcriptional regulator (Hong et al., 2008), this last observation suggests that it may belong to the family of proteins controlling gene expression at the transcriptional and post-transcriptional levels (Visa et al. 1996; Ladomery, 1997).

\section{Acknowledgements}

We would like to thank Kornelia Macha for discussion and help with some experiments and Alicja Obodczuk for help with manuscript preparation. 
This work was supported by the State Committee for Scientific Research (KBN) Grant 2P04B 01930.

\section{REFERENCES}

Cebrat M, Miazek A, Kisielow P (2005) Identification of a third evolutionarily conserved gene within the RAG locus and its RAG1-dependent and -independent regulation. Eur J Immunol 35: 2230-2238.

Cebrat M, Cebula A, Laszkiewicz A, Kasztura M, Miazek A, Kisielow P (2008) Mechanism of lymphocyte-specific inactivation of RAG-2 intragenic promoter of NWC: implications for epigenetic control of RAG locus. Mol Immunol 45: 2297-2306.

Hong M, Li W, Wang L, Jiang L, Liu L, Zhao H, Li Q (2008) Identification of a novel transcriptional repressor (HEPIS) that interacts with nsp-10 of SARS coronavirus. Viral Immunol 21: 153-161.

Kapranov P, Cheng J, Dike S, Nix DA, Duttagupta R, Willingham AT, Stadler PF, Hertel J, Hackermuller J, Hofacker IL, Bell I, Cheung E, Drenkow J, Dumais E, Patel S, Helt G, Ganesh M, Ghosh S, Piccolboni A, Sementchenko V, Tammana H, Gingeras TR (2007) RNA maps reveal new RNA classes and a possible function for pervasive transcription. Science 316, 1484-1488.
Kisielow P, Miazek A, Cebrat M (2008) NWC, a new gene within RAG locus: could it keep GOD under control? Int J Immunogenet 35: 395-399.

Kohler G, Milstein G (1975) Continuous cultures of fused cells secreting antibody of predefined specificity. $\mathrm{Na}$ ture 256: 495-497.

Ladomery M (1997) Multifunctional proteins suggest connection between transcriptional and post-transcriptional processes. Bioassays 19: 903-909.

Schatz DG, Baltimore D (2004) Uncovering the V(D)J recombinase. Cell 116: S103-S106.

Thompson CB (1995) New insights into V(D)J recombination and its role in the evolution of the immune system. Immunity 3: 531-539.

Visa N, Alzhanova-Ericsson AT, Sun X, Kiseleva E, Bjorkroth B, Wurtz T, Daneholt B (1996) A pre-mRNAbinding protein accompanies the RNA from the gene through the nucleoral pores and into polysomes. Cell 84: 253-264.

Wisniewski P, Master A, Kaminska B (2008) Cloning and purification of functionally active Fas ligand interfering protein (FIP) expressed in Escherichia coli. Acta Biochim Polon 55: 51-56.

Zhang W, Sloan-Lancaster J, Kitchen J, Trible RP, Samelson LE (1998) LAT: the ZAP-70 tyrosine kinase substrate that links $\mathrm{T}$ cell receptor to cellular activation. Cell 92: 83-92. 